\title{
Design of Hesitation Gestures for Nonverbal Human-Robot Negotiation of Conflicts
}

\author{
AJUNG MOON, McGill University, Canada \\ MANEEZHAY HASHMI and H. F. MACHIEL VAN DER LOOS, \\ University of British Columbia, Canada \\ ELIZABETH A. CROFT, Monash University, Australia \\ AUDE BILLARD, Ecole Polytechnique Fédérale de Lausanne (EPFL), Switzerland
}

\begin{abstract}
When the question of who should get access to a communal resource first is uncertain, people often negotiate via nonverbal communication to resolve the conflict. What should a robot be programmed to do when such conflicts arise in Human-Robot Interaction? The answer to this question varies depending on the context of the situation. Learning from how humans use hesitation gestures to negotiate a solution in such conflict situations, we present a human-inspired design of nonverbal hesitation gestures that can be used for Human-Robot Negotiation. We extracted characteristic features of such negotiative hesitations humans use, and subsequently designed a trajectory generator (Negotiative Hesitation Generator) that can re-create the features in robot responses to conflicts. Our human-subjects experiment demonstrates the efficacy of the designed robot behaviour against non-negotiative stopping behaviour of a robot. With positive results from our human-robot interaction experiment, we provide a validated trajectory generator with which one can explore the dynamics of human-robot nonverbal negotiation of resource conflicts.
\end{abstract}

CCS Concepts: • Human-centered computing $\rightarrow$ Collaborative interaction;

Additional Key Words and Phrases: Human-robot interaction, negotiation, hesitation, nonverbal communication

\section{ACM Reference format:}

Ajung Moon, Maneezhay Hashmi, H. F. Machiel Van der Loos, Elizabeth A. Croft, and Aude Billard. 2021. Design of Hesitation Gestures for Nonverbal Human-Robot Negotiation of Conflicts. ACM Trans. Hum.-Robot Interact. 10, 3, Article 24 (July 2021), 25 pages.

https://doi.org/10.1145/3418302

\section{INTRODUCTION}

In many everyday activities, it is common for interacting individuals to experience conflicts regarding priority to access shared resources. When the question of who should get access to the resource

This work has been supported by Natural Sciences and Engineering Council, Canada, and the EU project AlterEgo under Grant Agreement No. 600010.

Authors' addresses: A. Moon, Dept. of Electrical and Computer Engineering, McConnell Engineering Building, 3480 University Street, Room 541, Montreal, QC, Canada H3A 0E9; email: ajung.moon@mcgill.ca; M. Hashmi and H. F. M. Van der Loos, Dept. of Mechanical Engineering, The University of British Columbia, 2054-6250 Applied Science Lane, Vancouver BC, Canada V6T 1Z4; emails: maneezhay@gmail.com, vdl@mech.ubc.ca; E. A. Croft, Faculty of Engineering, Room 107, 14 Alliance Lane, Monash University, Clayton Victoria 3800 Australia; email: elizabeth.croft@monash.edu; A. Billard, Learning Algorithms and Systems Laboratory, EPFL STI I2S LASA ME A3 393 (Bâtiment ME), Station n ${ }^{\circ} 9 \mathrm{CH}-1015$ Lausanne, Switzerland; email: aude.billard@epfl.ch.

Permission to make digital or hard copies of all or part of this work for personal or classroom use is granted without fee provided that copies are not made or distributed for profit or commercial advantage and that copies bear this notice and the full citation on the first page. Copyrights for components of this work owned by others than ACM must be honored. Abstracting with credit is permitted. To copy otherwise, or republish, to post on servers or to redistribute to lists, requires prior specific permission and/or a fee. Request permissions from permissions@acm.org.

(C) 2021 Association for Computing Machinery.

2573-9522/2021/07-ART24 \$15.00

https://doi.org/10.1145/3418302

ACM Transactions on Human-Robot Interaction, Vol. 10, No. 3, Article 24. Publication date: July 2021. 
first is uncertain, people often nonverbally communicate with each other to negotiate a solution [3]. Humans typically express uncertainties through a variety of nonverbal cues - frowning, shrugging, putting our palms up, or exhibiting self-touch gestures, for example [15]. When we are uncertain or not sure what to do, we also hesitate to act, speak, or respond to a stimulus [35-37]. For example, when two people reach for the same object at the same time, they often manage to resolve the conflict by one yielding the object to the other or by claiming the object before the other obtains it [28]. When such a conflict occurs during a Human-Robot (HR) collaboration, what should a robot be programmed to do? Robots are typically programmed to stop moving or to yield at all times in resource conflict situations. While these are simple ways to ensure safety of the people interacting with the robot, they can also lead to suboptimal Human-Robot (HR) collaboration performance and experience. The overall time to completion of an HR collaborative task can be longer, and frequent and constant yielding behaviour of a robot can frustrate the collaborating human $[27,28]$.

As a practical and safe alternative, we propose to design robot behaviours that allow dynamic negotiation of an outcome with a person using nonverbal, negotiative hesitation gestures. We focus on an interactive scenario when the outcome (i.e., the question of who should access the shared resource first) is not predefined and requires negotiation. In this article, negotiation refers to the bidirectional interweaving of subplans between agents.

First, we conducted a Human-Human (HH) dyad experiment to collect and observe human negotiative hesitation gestures. Using a common trajectory pattern we observed from human hesitations, we created an artificial negotiative hesitation trajectory generator for a robotic system called the Negotiative Hesitation Generator (NHG). An online, video-based perception study, Study 2, was then conducted to validate the Negotiative Hesitation Generator (NHG). Results from this study provide empirical support that the NHG-generated trajectories are capable of producing robot behaviours that are perceived to be significantly more hesitant, persistent, animate, and anthropomorphic than a conflict response consisting of smoothly stopping and pausing.

The work presented in this article provides an empirically validated means of generating robot conflict responses that are perceived as hesitations by human observers, and paves the way to investigate HR negotiation of conflicts using purely nonverbal means.

\section{BACKGROUND}

Hesitations are social signals often observed as a form of disfluencies and uncertainties in human and animal behaviours. In Psychology, Doob [10] defines hesitation as the time that elapses between a stimulus and a response to the stimulus. Linguists, however, refer to disfluencies in speech, prolonged silence, or filled pauses, such as $u$ hs and ums, as hesitations [7, 23, 24, 39].

In Human-Robot Interaction (HRI), hesitation has been explored both as a behavioural indication of disfluencies experienced in HRI and as a means for robots to express uncertainties to humans. Bartneck et al., for example, measured how long it takes for a participant to turn off a robot while the robot pleaded to be left on [5]. The measured duration was seen as an indication of the participant's hesitation to follow through the experimenter's instruction in contrast to the robot's pleading. In contrast, Kazuaki et al. implemented intentional pauses between a person's demonstration of a task and a robot's performance of the demonstrated task [19]. This intentional use of pause was seen as a means to express the robot's degree of uncertainty about the demonstrated task. Others have explored the effectiveness of hesitation as a means to regulate turn-taking or expression of charisma in robot speech $[6,12]$. For instance, in the context of a verbal HRI, Chao and Thomaz implemented hesitations in robot behaviours as pauses that serve as precursors to a robot interrupting its turn to speak [6].

Other nonverbal expressions of hesitations have been implemented on a robot as well. In a robot-to-human handover task, Admoni et al. deliberately implemented delays in a robot's release 
of an object to a person [1]. The authors demonstrated that the strategic introduction of such delays in robot behaviour can have desirable effects, such as drawing a user's attention to the robot's gaze. In addition, Van den Brule et al. implemented head-scratching as a form of robot hesitation in a humanoid robot (Nao, Aldebaran Robotics) [38]. Through video-based and Wizard-of-Oz studies, the authors demonstrated how a robot that leads users to anticipate its poor performance is perceived more positively by the users.

While hesitation has often been measured as abnormally long pauses during interaction (e.g., Reference [34]), only a few scholars have studied kinematic hesitation gestures [8, 9, 27, 28, 30, 31, $37,40]$. This is partially due to the added complexity of processing kinematic hesitation signals, which have high dimensionality and are noisier than auditory pause signals.

One of the few studies that investigated kinematic hesitation gestures is a pilot study by Dondrup et al., which aimed to inform the design of spatial behaviour of mobile robotic platforms [9]. The authors sought to explore whether human hesitation behaviour can be captured in the observed motions of two people approaching and passing-by each other in a narrow corridor. They alluded that a person's hesitation behaviour upon encountering another person can be detected a drastic change in the person's velocity. With a focus on robotic manipulators, rather than mobile manipulators, our previous work also investigated the trajectory features of hesitations $[27,28]$. Within the context of HR collaborative assembly involving repetitive reach motions, we demonstrated that hesitations can be used as a communicative way for robots to respond to unexpected interruptions. In Moon et al. [28], we analyzed acceleration trajectories of the wrist in a subset of human hesitation gestures, called R-type hesitations, and formulated characteristic features from the trajectories as a series of cubic splines in the acceleration domain called the Acceleration-based Hesitation Profile (AHP). R-type hesitations involve the hesitating agent to immediately yield to the other agent upon encountering a resource conflict. This is in contrast with a previously unexplored, negotiative hesitations (formerly introduced as P-type in Moon et al. [27]), which involve the hesitating agents to pause upon encountering a conflict, and engage in a nonverbal dialogue-a back-and-forth dance of the hands mid-air by the two agents negotiating the conflict-until a resolution is reached. Negotiative hesitations are the focus of our investigation in this article. Using the Acceleration-based Hesitation Profile (AHP), we generated human-inspired R-type hesitation trajectories for a robot, which is the most basic type of human hesitation trajectories. Findings from Moon et al. [28] demonstrate that human observers correctly recognize the artificially generated robot-hesitation having the AHP as hesitations and can distinguish them from other similar robot motions. However, the type of hesitation behaviour (R-type) investigated in Moon et al. [28] was limited to the simplest form of hesitation behaviour, and one that is not meant to elicit nonverbal dialogue among the interacting HR pair. By definition, R-type hesitations are comprised of reacting to the conflict with a jerky halting motion followed by an immediate yielding of the resource before any dialogue can take place.

In developing a robotic system that can use hesitation gestures to resolve resource conflicts with humans interactively, it is important to equip the robot with a vocabulary of hesitation gestures that proactively engages the user in a nonverbal dialogue. One method of enriching the vocabulary of hesitation gestures for a robot is to understand and mimic how humans negotiate a solution to resource conflicts using hesitations (negotiative hesitations). Once a set of trajectory features is identified from negotiative human hesitations, these features can be used to generate analogous robot trajectories for HR negotiation of resource conflicts. The studies presented in this article contribute to this end by collecting negotiative hesitation motion samples from a human-subjects experiment and examining trajectory features that can be implemented in an HR collaboration context. 
One of the elements of negotiative hesitations that distinguish them from R-type hesitations is the expressed level of persistent interest and prolonged need for a resolution of the conflict at hand. By persistency, we refer to the quality that is expressed in the persistent state of an agent or the act of persisting in something (i.e., how persistent is an agent?). Persistency expressed as part of human behaviour has been studied in a variety of contexts [14, 16, 22]. Scholars in child development suggest that intentional communication-evidenced by persistence, among other behaviours-is a means for the communicator to manipulate the interlocutor rather than a mere attempt to engage in a conversation [17]. This suggests that negotiative hesitations by a robot that-in contrast to R-type hesitations-express a higher level of persistency are likely to be perceived by humans as intentional communication, possibly leading to the emergence of nonverbal dialogues

Persistence in communicative gestures is also closely related to negotiation behaviours. Golinkoff [16] states that persistence is seen as a proactive behaviour that is a necessary component of ongoing negotiation between mother and infant. An infant's persistent communication with the mother elicits a type of negotiation, which allows the infant to use communicative cues to manipulate its interlocutor/mother.

In this work, a level of persistency is assumed to be expressed in negotiative hesitations, since negotiative hesitations require the hesitating agent to have a degree of persistent interest in resolving a conflict. Therefore, the studies presented in this article explore persistency as a concept related to, but not trivially correlated with, hesitancy expressed in negotiative hesitations.

\section{STUDY 1: OBSERVING AND LABELLING HESITATIONS IN HUMAN-HUMAN DYADS}

To design robot hesitation behaviours that allow nonverbal negotiation of conflicts between a human and a robot, we first conducted a human-subjects experiment to collect a large set of naturally exhibited, negotiative human hesitations. The collected set of human behaviours was then labelled through a video-based online study using the Amazon Mechanical Turk platform [2]. This online study was designed to collect the varying degrees of perceived persistency and hesitancy people perceive from the recorded instances of hesitations. We outline the details of this process in this section.

The collected motion samples of hesitation gestures were then used to explore the common features that are present in the trajectories, thereby allowing us to design hesitation behaviours for a robot. The exploration of the trajectory data is presented in the following section (Section 4).

\subsection{Experimental Procedure}

Eight pairs ( $\mathrm{N}=16,3$ male-male, 2 female-female, 3 female-male pairs) of volunteers participated in this study in a lab environment. We used nine VICON cameras (six T40, two T160, and a synchronized digital camera) to capture participants' motion at $100 \mathrm{~Hz}$ ( $50 \mathrm{~Hz}$ for the digital camera). Both participants wore seven reflective markers on the joints of their dominant arm and hand (see Figure 1). This allowed the collection of Cartesian coordinates for each marker with respect to a predetermined reference point in space. Knowing the significant role wrist trajectories played in Moon et al. [28], participants wore two markers on either side of the wrist for more accurate measurement of this joint.

Participants stood facing each other across a table and played a card sorting game. On the table was a deck of randomly ordered cards split into two piles. In front of each participant were two aces of the same colour (either red or black). Starting from the two aces, the participants' task was to order two full sets of cards hierarchically (e.g., ace, 2, 3, and so on) according to an alternating colour pattern as fast as they can. 


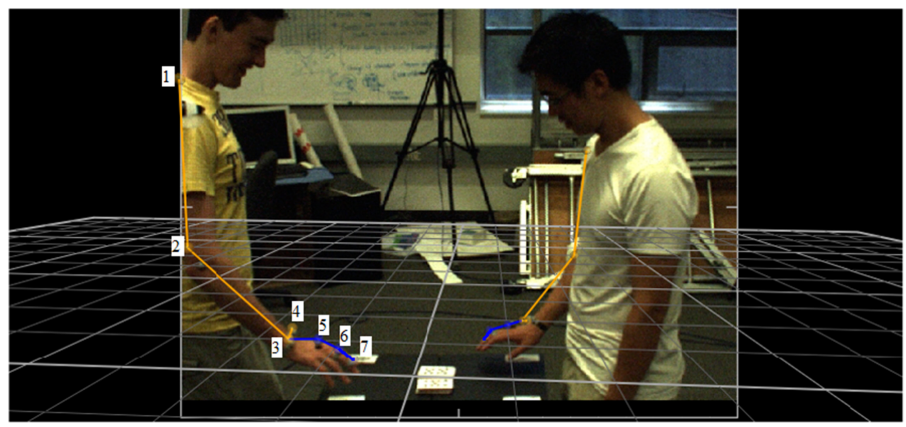

Fig. 1. A side view of the Human-Human Interaction (HHI) experiment captured from VICON's synchronized digital camera. Each pair of participants stood facing each other with a table between them and were asked to play a modified game of Solitaire. The numbers indicate the seven markers, used to track the participants' motion.

They were also instructed to access the two piles of cards in an alternating (left-right or rightleft) order and to grab or return only one card at a time. This requirement increased the cognitive load on the participants and prevented them from accurately keeping track of which pile of cards the other person would reach for next. By the design of the game, there were multiple natural occurrences of hesitations along with numerous reaching motions exhibited by each participant. Each pair of participants played the game twice, swapping the colour of the starting aces in the second round.

3.1.1 Labelling Human Hesitation Gestures. The experimenter watched all subject motions from the video recordings of the experiment and manually segmented and labelled participant motions that were deemed hesitations. In total, this qualitative process yielded 302 trajectories of hesitation gesture samples (171 from subjects on the left and 131 from the right side of the digital camera).

Subsequently, we conducted a video-based online survey to identify which of the 302 hesitation samples express lower or higher degrees of persistency and hesitancy. Participants for this online survey were recruited using the Amazon Mechanical Turk platform [2]. They were required to understand basic English to follow written instructions, and have good vision to be able to watch video recordings of human gestures.

Each video contained a segment identified as a hesitation plus a second before and after the motion segment used to provide the context of the motion. Each survey participant watched and scored a random selection of twenty hesitation videos on the following two seven-point scales:

- Persistency. "How persistent do you think the person on the LEFT is?" (1-Not persistent at all, 7-Extremely persistent)

- Hesitancy. "How hesitant do you think the person on the LEFT is?" (1-Not hesitant at all, 7-Extremely hesitant)

We changed the word LEFT to RIGHT, as appropriate, to highlight the specific individual of interest in the video.

\subsection{Results}

A total of 300 participants completed the online survey. For each segment of motion (video), approximately 20 individuals $(M=19.8, S D=3.25)$ provided both persistency and hesitancy scores. The average persistency and hesitancy scores were computed for each. The score averages range from 1.68 to $6.26(M=4.32)$ for hesitancy and 2.10 to $6.58(M=3.94)$ for persistency. The average 
standard deviation of the hesitancy and persistency scores range from 0.83 to $2.39(M=1.58)$ and 0.67 to $2.37(M=1.52)$, respectively. These are a reasonable range of scores and standard deviation considering the 7-point Likert scale data. The data collected from this study is used to analyze human motion data as discussed at length in the following sections.

Both the VICON study and the online survey were approved by the University of British Columbia Behavioural Research Ethics Board (H10-00503).

\section{EXPLORING HUMAN NEGOTIATIVE HESITATION TRAJECTORIES}

Our previous research [27, 28] demonstrated that there is a characteristic acceleration profile in R-type hesitations that is different from uninterrupted reach motions. Based on this characteristic, human-recognizable robot hesitations can be successfully generated even for a 7-Degrees-of-Freedom (DoF) robot (e.g., WAM, Barrett Technologies, Cambridge, MA) with a non-anthropomorphic morphology [28]. However, the same research showed that the same trajectory features are absent in negotiative hesitations (formerly introduced as P-type in Moon et al. [27]), and that the acceleration profiles of negotiative hesitations collected in the study are similar to that of uninterrupted reaches. Nonetheless, the survey results of Study 1 suggest that humans can perceive and recognize the subtle differences between the two types of motions. This suggests that negotiative hesitation trajectories must be characterized in a different domain or using a different set of trajectory features.

Pre-processing. To characterise negotiative hesitation trajectories, we first transformed the recorded Cartesian coordinates of the joints with respect to the coordinates of the shoulder. We computed the average of the two markers placed on either side of each participant's wrist for a more consistent treatment of the wrist joint measurement. The trajectories were filtered using a Butterworth low-pass filter at the cutoff frequency of $10 \mathrm{~Hz}$, the highest frequency at which voluntary human motion can occur.

Segmentation. Coordinates of practical target locations for each participant were computed, such that one segment of a reach motion starts at the beginning of a motion (zero velocity crossing) and ends at the Cartesian points in space the person tried to reach for $\left(T_{m}\right.$ for the main, and $T_{p}$ for the partner participant), based on his/her preferred way of taking cards from the deck. $T_{m}$ and $T_{p}$ were typically near one of the four corners of the card. Since it was not clear which patterns exist in marking the start and end of a hesitation, these samples were manually segmented by watching the video recordings.

\subsection{Data Exploration}

After excluding outliers, ${ }^{1}$ we had a total of 1,706 reach and 298 hesitation motion segments available for analysis. After a number of failed analyses using trajectories of all joints, we also narrowed the focus of the analyses to the wrist joint. Herein, $M(t)$ and $P(t)$ refer to the Cartesian coordinates of the main and partner participants' wrist trajectories, respectively. The main participant is the one whose motion segment is labelled as a reach or a hesitation. The partner participant's trajectory refers to whatever motion the partner was exhibiting during the main participant's reach or hesitation.

We investigated a set of 75 features from the segmented sample trajectories of both hesitation and reach motions. This initial set includes the five types of features-maximum (max), minimum $(\min )$, mean $(\mu)$, amplitude $(A)$, and the number of zero crossings $(\rho)$-computed for the following fifteen metrics:

\footnotetext{
${ }^{1}$ Samples with $\geq 3$ SD in velocity maximum and range.
} 

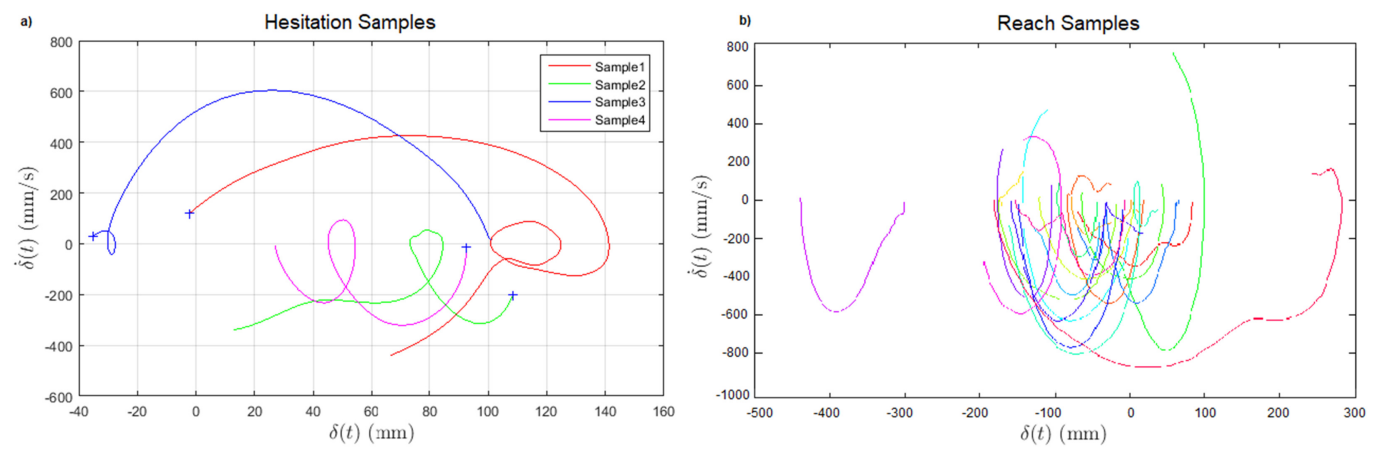

Fig. 2. (a) Overlay of hesitation samples demonstrating hesitation loops in the $\dot{\delta}(t)$ vs. $\delta(t)$ state space that encircle the zero $\dot{\delta}(t)$ axis. (b) Overlay of reach samples in $\delta(t)$ vs. $\dot{\delta}(t)$ state space that demonstrates the absence of circular loops around $\dot{\delta}(t)=0$.

- $d_{1}(t)=\left\|M(t)-T_{m}\right\|$, the main participant's Euclidean distance to the target, and its first $\left(\dot{d}_{1}(t)\right)$ and second derivative $\left(\ddot{d}_{1}(t)\right)$,

- $\alpha_{1}(t)$, the main participant's principal component of the segment along the direction of travel, and its first $\left(\dot{\alpha}_{1}(t)\right)$ and second derivative $\left(\ddot{\alpha}_{1}(t)\right)$,

- $d_{2}(t)=\left\|P(t)-T_{p}\right\|$, the partner participant's Euclidean distance to the same target, and its first $\left(\dot{d}_{2}(t)\right)$ and second derivative $\left(\ddot{d}_{2}(t)\right)$,

- $\alpha_{2}(t)$, the partner participant's principal component of the motion segment along the direction of travel, and its first $\left(\dot{\alpha}_{2}(t)\right)$ and second derivative $\left(\ddot{\alpha}_{2}(t)\right)$,

- $\delta(t)=\left\|M(t)-T_{m}\right\|-\left\|P(t)-T_{p}\right\|$, the difference between the main and partner participants' Euclidean distances to their respective target locations, and its first $(\dot{\delta}(t))$ and second derivative $(\ddot{\delta}(t))$.

Upon the exploratory analysis using inferential statistics, we identified that trajectory metrics $\dot{d}_{1}(t)$ and $\dot{\delta}(t)$ are the most promising is helping to characterize hesitation motions. The procedure included the generation of a regularized regression using a shooting algorithm, for example, to identify a subset of trajectory features that are key to predicting the expressed level of hesitancy in human hesitation gestures. A detailed description of our exploratory procedure leading to this conclusion is outlined in Reference [26].

We find that when hesitation samples are plotted in the state space of $\dot{\delta}(t)$ and $\delta(t)$, the trajectories tend to have the shape of a loop (see Figure 2(a)). This is in contrast to reach trajectories visualized in the same state space that does not have such loops, as shown in Figure 2(b).

\subsection{Characterising Hesitation Loops}

To verify whether the loops in the $\dot{\delta}(t)$ and $\delta(t)$ state space are indeed characteristic of negotiative hesitation motions in contrast to reach motions, we counted the number of samples that have a looped shaped in the state space. Results show that a total of 134 hesitation samples (approximately $45 \%$ of total hesitation samples) share this looped trajectory that is absent in reach gestures. Further analysis of the location and direction of the loops suggests that all of the loops in hesitation samples encircle $\dot{\delta}(t)=0$. Due to the linear relationship between $\dot{\delta}(t)$ and $\dot{d}_{1}(t)$, these loops can also be observed in $\dot{d}_{1}(t)$ vs. $\delta(t)$ space.

With the sample hesitations that have these loops, we computed the Euclidean distance travelled by the main participant between the two zero crossings of the encirclement. This distance 


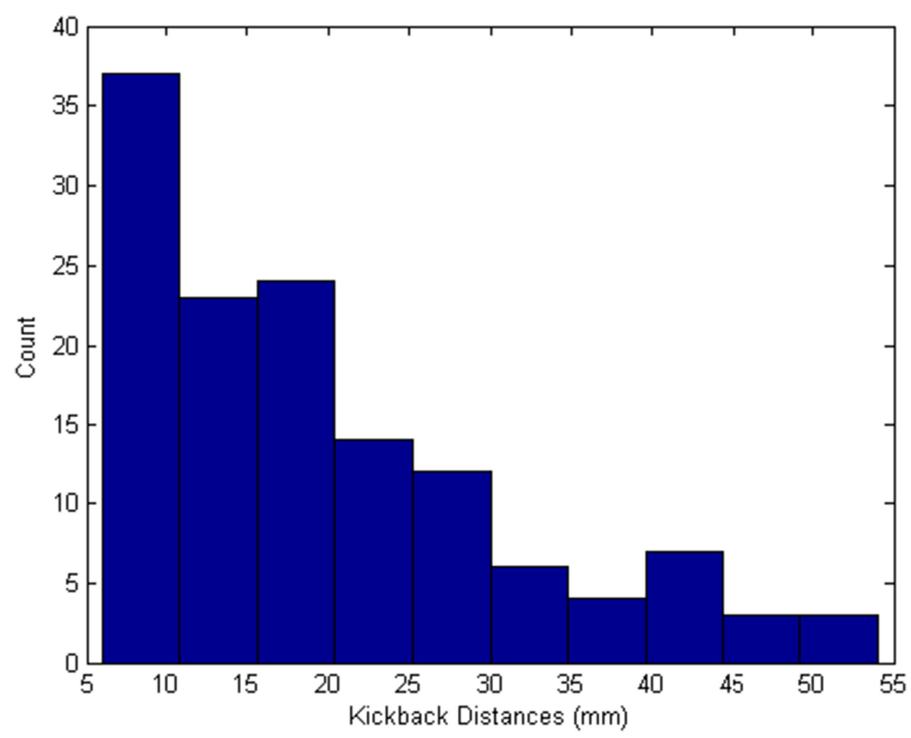

Fig. 3. Distribution of KD collected from the 134 samples of hesitations that encircle $\dot{\delta}(t)=0$.

represents how much the main participant retracted, if any, while the negotiative hesitation took place. Based on the computation, the distribution of this distance, Kickback Distance (KD), has a mean of $19.6 \mathrm{~mm}(S D=11.8)$. Note that, as shown in Figure 3, the distribution of Kickback Distance $(\mathrm{KD})$ of human hesitations is non-normal with most of the values occurring between 5 to $30 \mathrm{~mm}$. It is possible that we may lack the number of samples to make general claims about whether all KD values in human hesitations are non-normal.

It is important to note that, since behaviours humans perceive as hesitations can take on many different forms, including lack of motion, the characteristic loops referred to as hesitation loops in this section are not proposed as a feature that is present in all hesitations. Instead, they are presented as characteristic features of a subset of hesitation behaviours studied in this work, and put forth as components that can be implemented onto a robot to generate human recognizable artificial hesitation behaviours.

The ultimate goal of having such dynamic and reactive behaviours in a robot is to be able to generate negotiative behaviours in HRI that result in an interactive resolution of conflicts. To this end, hesitation loops are discussed in more detail in the following section and are used to frame the design of the NHG.

\subsection{The Four Cases of Hesitation Loops}

Given the discovery of hesitation loops, one can formulate an intuitive understanding of the context embedded in the trajectory in state space.

First, trajectories that move in the positive direction of state space, or crossing $\delta(t)=0$ from the left to the right quadrants, can indicate that the main participant was moving toward the target or the partner was moving away from the target. In the former case, the trajectory must travel in the upper quadrants $(\dot{\delta}(t)>0)^{2}$; in the latter case, the trajectory must travel in the lower quadrants

$\overline{{ }^{2} \dot{d}_{1}(t)>\dot{d}_{2}(t)}$. 

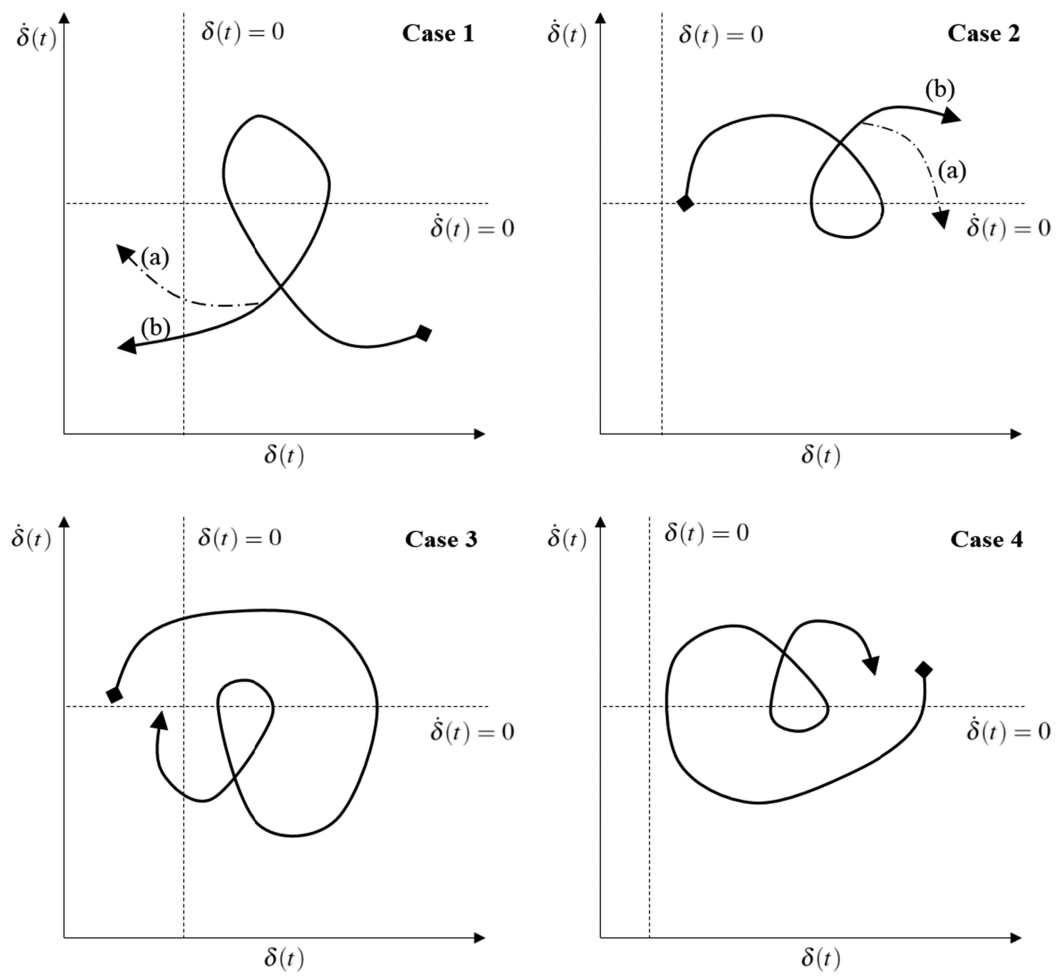

Fig. 4. The four cases of hesitation loops demonstrated in the $\dot{\delta}(t)$ vs. $\delta(t)$ state space, where $\delta(t)=$ $\left\|M(t)-T_{m}\right\|-\left\|P(t)-T_{p}\right\|$. Each case represents a spatial context between the two interacting agents' motions as outlined in Section 4.3. All hesitations loop around $\dot{\delta}(t)=0$. The start and end points of the arrows indicate the starting and ending states of the agents' motions, respectively. Any state to the left $\delta(t)=0$ indicates that the partner is farther from the target than the main participant, whereas states to the right of $\delta(t)=0$ indicate that the partner is closer to the target than the main participant. States above $\dot{\delta}(t)=0$ indicate that the speed of the main participant is faster with respect to the partner participant; states below $\dot{\delta}(t)=0$ indicate that the speed of the partnering participant is faster than that of the main participant.

$(\dot{\delta}(t)<0){ }^{3}$ Since hesitation motions of the main participant are of focus, only the former case is interesting for our discussion.

Trajectories that move in the negative direction in the state space represent either that the partner participants were getting closer to the target than the main participant, or that the main participant was moving away from the target while the partner remained stationary. Likewise, only the trajectories that move from right to left while the partner is moving faster than the main participant $(\dot{\delta}(t)<0)^{4}$ are relevant to the discussion of hesitations.

There are four cases of observed hesitations that describe the spatial contexts in which hesitations occur. Figure 4 provides a visual demonstration of the cases. This section outlines the Human-Human $(\mathrm{HH})$ dyad's interaction dynamics that are represented in these cases and what the entering and exiting directions of a hesitation loop mean in these contexts:

\footnotetext{
$\overline{{ }^{3} 0-\dot{d}_{2}(t)<0}$.

${ }^{4} \dot{d}_{1}(t)<\dot{d}_{2}(t)$.
} 
Case 1. Hesitation samples, in this case, start with a state of $(\delta(t)>0, \dot{\delta}(t)<0)$. That is, the main participant is farther away from the target and moving slower than the partner at the onset of a hesitation $\left(d_{1}(t)>d_{2}(t)\right)$. The main participant closes the distance gap as he/she gains speed and catches up to the speed of the partner (crosses $\dot{\delta}(t)=0$ in the upward direction). The main participant is briefly faster than the partner, but quickly slows down with respect to the partner (crosses $\dot{\delta}(t)=0$ in the downward direction). In some of the cases, the main participant immediately speeds up again with respect to the partner, suggestive of the main participant's second attempt at accessing the target (see Case 1 (a) in Figure 4). In other cases, the main participant continues to slow down, yielding to the partner (see Case 1 (b) in Figure 4).

Case 2. In this case, hesitation samples start with a state of $(\delta(t)<0, \dot{\delta}(t) \leq 0)$. The value of $\dot{\delta}(t)$ is near zero, indicating that the partner participant may be engaged in some other activity (e.g., shuffling cards sideways on his/her side of the table) or at rest when the main participant starts his/her reach (i.e., $\dot{\delta}(t)>0$ ). In the example depicted in Figure 4, the main and the partner participants are almost equidistant to the target at the onset of the main participant's motion. After the main participant is moving faster and is closer to the target, the partner starts to move towards the shared target. Either by a brief acceleration of the partner or deceleration of the main participant's motion (hesitation), the partner moves faster toward the target before the main participant re-attempts to continue his/her reach. The participants may go through multiple cycles in which one of the participants moves slower than the other, crossing $\dot{\delta}(t)=0$ downward (see Figure 4 Case 2(a)). Alternatively, the partner may yield to the main participant (see Figure 4 Case 2(b)). Going through multiple cycles of the trajectory pattern is indicative of multiple re-attempts-referred to as Re-attempts (RA)-by the main participant to gain access to the target resource.

Case 3. Cases 3 is a variation of Cases 1 described above. In Case 3, the main participant reaches for the target, but the speed toward, and subsequently, the distance to the target is overtaken by the partner who moves faster-perhaps despite having started his/her reach later than the main participant. This dynamic brings the pair's trajectory to cross the $\dot{\delta}(t)=0$ state downwards once before crossing it again in the manner described in Case 1.

Case 4. Likewise, Case 4 is a variant of Case 2, where only the starting state of the dyad is different. The example Case 4 illustrated in Figure 4 shows a starting state where the main participant is farther away from the target than the partner. While the main participant is moving faster toward the target in the beginning, this is overtaken by the acceleration of the partner participant until the two agents are approximately equidistant from the target. Their speed and respective distances to the target lead them to eventually go through the same hesitation loop as described in Case 2 afterwards.

Understandably, because $\delta(t)$ and $\dot{\delta}(t)$ combine the effects of both the main and the partner participants, the zero crossings of the system depend on both agents' actions. Hence, the generation of hesitation loops in HRI requires a translation of this trajectory pattern into a system in which only one of the agents' (robot's) motions can be precisely controlled-needless to say, motions of the other agent (human's) remain outside of our control. For the purpose of developing a trajectory generator that can recreate such hesitation dynamics in HRI, one can detect when such encirclement of $\dot{\delta}(t)=0$, such as detection of the zero crossings, occurs and manipulate 
the robot's reaching motion to produce the kickback motion (move backwards by a Kickback Distance (KD)) that completes the loop. Herein, a $\dot{\delta}(t)=0$ occurrence is referred to as the Trigger State (TS) to designate the state at which a hesitation behaviour of a robot is to be triggered.

\section{DESIGN OF THE NEGOTIATIVE HESITATION GENERATOR}

To investigate the efficacy of using negotiative hesitations in HRI as a mode of resolving resource conflicts, interactive behaviours of a robot must be able to generate the elements of human hesitation behaviours mentioned in the previous section. This artificial hesitation behaviour must also be implemented on a robot in such a way that the system can respond to human hesitations in real-time to allow negotiative nonverbal dialogues to emerge.

Among other alternatives, we chose to use the Linear Dynamical System (LDS) approach to generate reference trajectories of artificial hesitations. A Linear Dynamical System (LDS) is defined as the following:

$$
\dot{x}=A x+b .
$$

In this differential equation, $A$ is a matrix and $b$ is a vector describing a vector field in the designated state space. These parameters represent the linear dynamics that exist between the state variable, $x \in \mathbb{R}^{d}$, and the rate of change expressed in $\dot{x}$. Hence, iterative computation of the differential equation results in the determination of the trajectory of the linear system through the state space. In robotics, $x$ typically represents unambiguous states of a robot, such as joint angles. Therefore, computed states and state velocities can be used as reference trajectories with which to control a robot.

One of the reasons for choosing to implement artificial hesitation behaviours with an LDS is that the Dynamical System (DS) approach offers reference trajectory generators that are reactive, real-time, and robust to disturbances or changes to the environment. In contrast, our previous work used trajectories generated using splines [28]. The splines function as an interpolation between two boundary states defined early in the motion trajectory. Hence, unexpected disturbances that push the robot out of its planned trajectory, for example, typically result in an undesirable, highjerk movement of the robotic platform. This happens as the system tries to catch up to larger than the planned difference between the state that has been planned for its next time step and the current, disturbed state of the robot. In comparison, trajectories that are generated using a Dynamical System (DS) iteratively uses the robot's current state for the computation of the desired next state. With a DS that converges to a target location, any sudden disturbances to the system only change the value of the input state used to compute the desired next state of the system. Since the reference state for the next time step is computed based on the vector field, sudden changes to its input states are seamlessly handled. This highly reactive nature of DS-based systems has also been showcased in complex real-time obstacle avoidance tasks, as well as tasks that involve catching flying target objects $[21,25]$.

With the development of algorithms that can guarantee the stability of a DS during the training phase of the system, such as Stable Estimator of Dynamical Systems [20], the DS has also been used to encode a variety of tasks for a robot as a teach-by-demonstration method [11, 18, 32]. Given its potential to develop a large vocabulary of activities for robots to perform, implementing hesitation behaviours on a DS opens up the possibility for artificial hesitation behaviours to be triggered during tasks not envisioned or tested in this article. With this in mind, we designed a DS-based trajectory generator that takes into consideration point-to-point motions involved in reaching for a shared resource. Such motions are not only foundational to many tasks, but they are also the main type of motion used in the type of resource conflicts discussed in this article.

Coincidentally, implementation of the Trigger State (TS)-the state at which the controller should trigger a hesitation behaviour-and KD-the distance to retract as part of the hesitation 

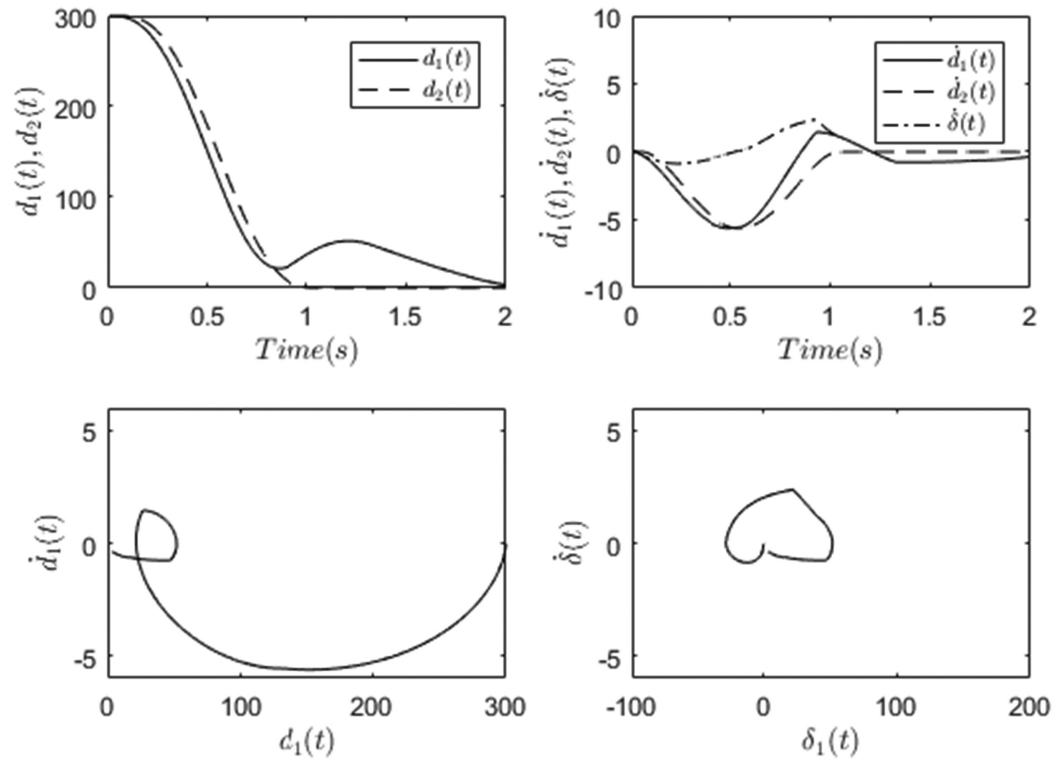

Fig. 5. Simulation output demonstrating the NHG implementation using quintic splines. Two quintic splines are generated to represent reach motions of two agents reaching for the shared target a fraction of a second (0.05 s) apart from each other. The main agent's quintic spline is interrupted when a TS is detected. This triggers the main agent to move back by KD before reaching for the target again. This results in a circular motion in the $\delta(t)$ state space (representing the difference between the main and the partner participant's distance to their respective target locations) around $(0,0)$ of $\dot{\delta}(t)=0$.

behaviour, determined experimentally-in an LDS yields a simple control architecture. As discussed in the previous section, for hesitation loops to be generated in an HRI, one must remember that only one of the agents, the robot, is within the scope of our control. Moreover, $\delta(t)$ and $\dot{\delta}(t)$ represent ambiguous states that can be translated into an infinite combination of states in the physical world. ${ }^{5}$ Therefore, rather than devising a control signal in $\delta(t)$ and $\dot{\delta}(t)$ space, one can monitor the state space to detect the TS in which an encirclement of the $\dot{\delta}(t)=0$ is desired.

To do this, we employed a simple LDS in $d_{1}(t)$ vs. $\dot{d}_{1}(t)$ space $\left(\dot{d}_{1}(t)=A d_{1}(t)\right)$ that can be used to reach for a target, and implemented an upper layer module that monitors for the TS in $\delta(t)$ vs. $\dot{\delta}(t)$ state space. Knowing the location of the shared target resource, $T$, the values of $d_{1}(t)$ and $\dot{d}_{1}(t)$ can be translated to Cartesian positions and velocities along a desired path of motion. This transforms the ambiguous one-dimensional states, $d_{1}(t)$ and $\dot{d}_{1}(t)$, to the unambiguous position and velocity $M(t)$ and $\nabla M(t)$.

Once a TS is detected, the upper layer algorithm moves the equilibrium point, or the point of convergence, of the LDS by a KD. The devised system that produces reference trajectories using this control regime is the Negotiative Hesitation Generator (NHG). To demonstrate that such a layered approach produces the desired encirclement of $\dot{\delta}(t)=0$, this regime was simulated in MATLAB (The MathWorks Inc., Natick, MA) with quintic splines that represent two agents reaching for the same target. As shown in Figure 5, the generated reference trajectory from the simulation encircles the $\dot{\delta}(t)=0$ observed from HH negotiative hesitations. The upper layer module was simulated using splines, since this was practical due to the off-line nature of the

\footnotetext{
${ }^{5} \delta(t)$ and $\dot{\delta}(t)$ are functions of relative Euclidean distances to the target. Hence, $\delta(t)=2 \mathrm{~cm}$ can mean one agent being anywhere in a $2 \mathrm{~cm}$ radius from the target and the other being $4 \mathrm{~cm}$ away.
} 
simulation. A spline-based implementation of the TS and KD for real-time, in-person HRI will require added complexities in the control architecture to ensure responsiveness of the system without generating undesirable, residual motion. The system architecture to implement the NHG using a LDS is described in detail in Section 6.2.

\section{STUDY 2: VALIDATING THE NEGOTIATIVE HESITATION GENERATOR}

In this section, we outline an online study we conducted to (a) demonstrate that the robot motions resulting from the NHG can communicate hesitation to third party observers, and (b) identify values of KD and Re-attempts (RA) that can be used for effective implementation of the NHG.

As outlined in Section 5, a premise of the NHG is in the presence of hesitation loops found in the $\dot{\delta}(t)$ vs. $\delta(t)$ state space. When the state space is interpreted with the context of an inperson HRI, it allows one to understand the dynamic, spatial interaction between the two agents. In this state space, the NHG calls for the implementation of the following three elements: TS, a state at which human agents tend to hesitate in response to an imminent resource conflict; KD, the amount of distance the hesitating agent retracts as part of the hesitation behaviour before reattempting to access the resource in conflict; and RA, the maximum number of times the robot should re-attempt to access the conflicted resource before yielding. While TS refers to a state at which hesitation behaviour should be triggered, implementation of an NHG involves assigning values of $\mathrm{KD}$ and $\mathrm{RA}$, both of which are adjustable parameters. In the $\mathrm{HH}$ hesitation samples collected in Study 1 (Section 3), a range of values of both KD and RA were observed. Hence, within the range of observed parameter values, it is useful to investigate what pairs of values for these parameters are acceptable for the NHG to generate artificial robot motions that convey a state of hesitation.

In this section, we evaluate the efficacy of a set of KD and RA parameter values against each other, in addition to a smooth stopping motion typically implemented in robotic collision avoidance behaviours. We hypothesized the following:

- Hypothesis 1. Robot hesitation responses generated by the NHG are perceived to be more hesitant and persistent than a smooth stopping behaviour.

- Hypothesis 2. Robot hesitation responses generated by the NHG are perceived to be more animate and anthropomorphic than a smooth stopping behaviour.

- Hypothesis 3. Considering the fact that robot hesitation responses generated by the NHG lead to the robot re-attempting to reach the target rather than yielding right away, they are perceived to be more dominant and useful than a smooth stopping behaviour.

- Hypothesis 4. Robot hesitation responses generated with a human-inspired kickback distance $(0 \leq K D<19 \mathrm{~mm})$ are perceived to be more hesitant than those with $\mathrm{KD}$ outside this range.

- Hypothesis 5. Robot hesitation responses showing a larger number of RA are perceived to be more persistent, dominant and useful than those with a larger RA.

Obtaining empirical support for these hypotheses would validate the use of the trajectory generator as a mechanism that is adequate for creating artificial robot hesitation responses for in-person interactions with humans. In addition, it would provide a measure of the dominance, animacy, and anthropomorphic qualities perceived by human observers in the generated trajectories. These perception measures provide valuable information to consider in implementing robot behaviours for an improved HRI experience. The remainder of this section outlines the experimental procedure used to conduct the online survey (Section 6.1), the technical system used to implement the NHG (Section 6.2), and empirical and qualitative findings from the analysis (Section 6.4). Section 7 discusses the implications of the findings in detail. 


\subsection{Experimental Procedure}

This online survey-based study was structured to be similar to Study 1 . The participants were recruited via Amazon's Mechanical Turk system to watch and report on their impression of a series of videos. All survey participants gave consent online by explicitly selecting the option "I consent to participate." Upon giving consent, participants provided their demographical information (age and gender). Afterward, participants were asked to watch a short introductory video before proceeding with the survey. This introductory video was made to provide the context of the interaction that the participants need to know to understand the videos that followed. The introductory video showed the experimenter and the robot, facing each other and collaborating on an assembly task. In front of the robot were two liquid pumps, and between the two agents was a dispenser operated with a button on top. Near the experimenter was a bin containing uncooked lentil beans of two different colours. The following text appeared at the bottom of the screen:

You will be watching a person and a robot working on a collaborative task. In this task, the person sorts and places items onto the dispenser in the centre. Meanwhile, the robot helps by handling the liquid pumps, and flushing the dispenser using the black button. Occasionally, the person and the robot reach for the dispenser at the same time.

At the end of the introductory video showed the experimenter and the robot reaching for the same dispenser at the same time, demonstrating a conflict of a resource. Near the end of the video, the video showed the robot and the experimenter reaching for the dispenser at the same time. See Figure 6 for a screen capture of this segment of the introductory video. The video faded to black before revealing who got the right of way. The video ended without showing the resolution of the conflict (i.e., who gets access to the resource). The behaviour of the experimenter remained the same in all videos.

In the following pages of the survey, participants watched a total of 15 videos. All videos started with the motion of the robot returning from the dispenser after successfully having reached and pressed it. Afterward, each of the videos showed the robot pressing on one of the liquid pumps, then reaching for the dispenser again. But this time, the experimenter reached for the dispenser as well, creating a conflict of resource. The videos showed the robot responding to the conflict with either a smooth stopping behaviour, or a motion generated by the NHG with one of the fourteen parameter pairs (see Figure 7 for the full list of conditions). For the purpose of testing Hypothesis 4, values $0,10,19$, and $40 \mathrm{~mm}$ are used to represent the range of $\mathrm{KD}$ observed from Study 2. The KD value of $19 \mathrm{~mm}$ represents an approximate average of the distribution of $\mathrm{KD}$ observed in human hesitation behaviours. The KD values 0 and 40 represent the two extremes of the distribution.

Ideally, the parameter pair would include five recordings for each value of $\mathrm{KD}$, spanning $\mathrm{RA}$ values from 0 to 4 . However, videos for the parameter set $(K D=10, R A=4)$ and $(K D=40, R A=$ 0 ) were lost from our recording due to a technical failure. They could not be re-recorded due to logistical constraints of international collaborative research. ${ }^{6}$ In addition, the KD value of 0 inherently does not provide any back and forth motion, rendering the value of RA meaningless. Given the lack of re-attempt behaviour, the robot to quickly retreat to its home position as soon as an imminent conflict is detected. The parameter set $(K D=0, R A=0)$ serves as a reference point to study the main effects of $\mathrm{KD}$ and RA as is explained in more detail in the results section

\footnotetext{
${ }^{6}$ All of the videos were recorded at Ecole Polytechnique Fédérale de Lausanne (EPFL) at a location and with an experimental setup that is no longer accessible to us.
} 


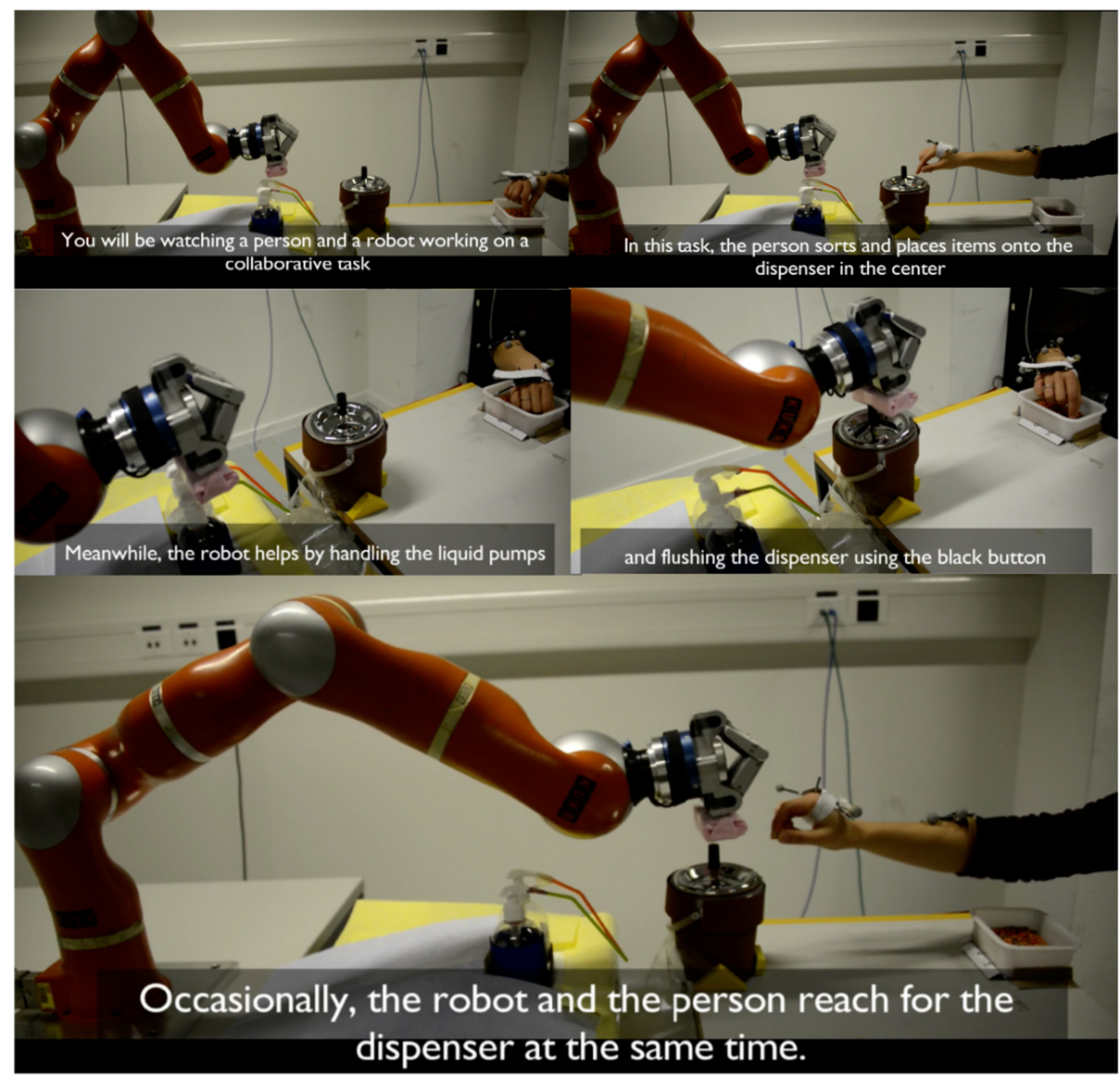

Fig. 6. A screen capture of the introductory video shown to participants in Study 2. All other nonintroductory videos shown to the participants were captured from the same camera angle, showing only the experimenter's arm, the robot, and the experimental setup.

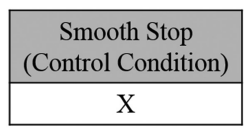

\begin{tabular}{|c|c|c|c|c|c|c|}
\hline \multicolumn{7}{|c|}{ The NHG Conditions } \\
\hline \multicolumn{2}{|c|}{} & \multicolumn{5}{|c|}{ RA } \\
\cline { 2 - 7 } \multicolumn{2}{|c|}{} & 0 & 1 & 2 & 3 & 4 \\
\hline \multirow{5}{*}{ KD } & 0 & $\mathrm{X}$ & & & & \\
\cline { 2 - 7 } & 10 & $\mathrm{X}$ & $\mathrm{X}$ & $\mathrm{X}$ & $\mathrm{X}$ & \\
\cline { 2 - 7 } & 19 & $\mathrm{X}$ & $\mathrm{X}$ & $\mathrm{X}$ & $\mathrm{X}$ & $\mathrm{X}$ \\
\cline { 2 - 7 } & 40 & & $\mathrm{X}$ & $\mathrm{X}$ & $\mathrm{X}$ & $\mathrm{X}$ \\
\hline
\end{tabular}

Fig. 7. Outline of the conditions tested for Study 2. The conditions tests are marked with an "X."

(Section 6.4). The smooth stop response is used as a control condition with which to contrast all NHG-based trajectories.

Presented below each video were a set of questions that echo the questionnaire used in Study 1. The first nine questions were designed to collect six self-report measures: three questions for measuring Anthropomorphism and Animacy, derived from the Godspeed Questionnaire [4]; four 
questions for measuring Dominance and Usefulness, two perceived team measures from Reference [29]; and Hesitancy and Persistency measures. ${ }^{\text {? }}$

Findings of Study 1 suggest that responses to Persistency and Hesitancy may not be enough to describe the quality of motion the participants pay attention to. Therefore, a textbox was included to collect optional, open-ended qualitative responses to the question "What adjectives would you use to describe the motion of the robot observed in the video? (Optional)."

Participants could replay the video as many times as they liked to complete the survey. Each video was 16 to $22 \mathrm{~s}$ in length. All participants watched all fifteen videos in random order. Participants were offered a financial reward of \$1.60 USD for completing the survey.

\subsection{Technical Implementation}

The experimental system consisted of a 7-degree-of-freedom (DoF) KUKA LWR 4+ (KUKA Robot Group, Augsburg, Germany) manipulator controlled using the Fast Research Interface (FRI) at a $1 \mathrm{kHz}$ frequency, and an OptiTrack (NaturalPoint Inc. DBA OptiTrack, Corvallis, OR) motion capture system operating at a $120 \mathrm{~Hz}$ sampling rate. The motion capture system was used to track markers on the experimenter's hand and forearm.

In all of the conditions, the robot's task involved reaching and retraction motions to and from the two liquid pumps and the dispenser button. Depending on the experimental condition, the robot used either a Hermite quintic spline or the NHG to generate the reaching motion to and from the dispenser as further described below. For all conditions, once the robot reached the dispenser button or one of the pumps, it was programmed to press on the button/pump using a Hermite quintic spline. This ensured that all aspects of the generated robot motions remain equal for all conditions except for the reaching motion and the robot's response to conflicts.

In the fourteen conditions that involve values of KD and RA, the LDS implementation of the NHG was used to generate reference Cartesian position trajectories of reaching motions. The robot was programmed to re-attempt (i.e., reach for the resource again immediately after returning to the starting position) a maximum of four times before giving up its access to the resource and resuming its task of tending to the liquid bottles. ${ }^{8}$

In the Smooth Stop (control) condition, the robot's reaching motion was generated using a Hermite quintic spline. The spline-based trajectory generator was designed to smoothly stop and pause the robot for $1.0 \mathrm{~s}$, wherever the robot might be at the time and wherever the conflict was detected.

This technique takes the start and end states of the robot and the desired travel completion time to interpolate between the two points in a minimum-jerk manner. The resulting trajectory allows for the robot to trace smooth reference trajectories considered humanlike [13]. When the robot detects a conflict near the onset of its reach motion, it exhibited the R-type hesitation using the AHP. When the conflict was detected after a time window, it was programmed to remain at its current location, leading it to come to a full stop and hold its position for 1 second before retreating. Since this condition does not employ the notion of the TS used in the NHG to determine the state at which the robot should trigger a conflict response behaviour, the system was programmed to use proximity and direction of motion between the robot and the experimenter to detect possible HR resource conflict states. When the robot and the experimenter moved toward each other and were closer than $5 \mathrm{~cm}$ from each other, the robot was programmed to remain at the current location

\footnotetext{
${ }^{7}$ The Hesitancy and Persistency measures were used in Study 1 with word pairs (Hesitant-Not hesitant) and (Persistent-Not persistent). The same word pairs are used in this study.

${ }^{8} \mathrm{~A}$ video recording of the generated robot hesitation triggered and an experimenter is available online https://youtu.be/ b7w8Gt09fOU.
} 
such that it comes to a full stop. After $1 \mathrm{~s}$ of pause in the stopped state, the robot retracted back to its home position.

6.2.1 System Architecture. The system architecture employed for this study was implemented in Robot Operating System (ROS) [33]. Three main components were custom developed for this experiment:

(1) a communication node dedicated to receiving, transforming, and publishing sensed information from OptiTrack,

(2) an action server that registers and maps requested actions from action clients onto a set of predefined action policies that ultimately generate desired reference trajectories, and

(3) a client action node that acts as the master script to trigger different robot actions at appropriate times.

First, the data stream from OptiTrack was broadcast through a local area network and converted into a data format appropriate for further use of the data in Robot Operating System (ROS). The ROS action client-server protocol was employed to interface the trajectory generator algorithms with the KUKA FRI interface controlling the robot. The experiment's main action client acted, in part, as a server for smaller action clients. The main client is responsible for the continuous monitoring of human motion captured via OptiTrack. When initialized, it launches a dedicated node to receive and convert the stream of OptiTrack data into a local coordinate system as well as Euclidean distance to the shared resource (dispenser). The final Rostopic containing human motion information is ultimately communicated to the action server to trigger different trajectory responses for the robot. The smaller action clients trigger various behaviours, such as reach or retract, to the main client, which in turn requests actions to be serviced by the action server. The action server, upon receiving a request, generated and communicated the reference trajectories for the control interface to actuate the robot.

To generate a negotiative hesitation response, we first used the same quintic trajectory generator as in the smooth stop condition for reach toward the target. Upon detection of conflict, the trajectory generator of the robot was overwritten by the NHG, an LDS implementation of the characteristics empirically observed in human hesitations was employed. The LDS was tuned such that one round of reach, press, and retract motion took $3.2 \mathrm{~s}$, matching the time it takes the robot to complete the same action in the smooth stop condition. This was to ensure that the only difference between the smooth stop and hesitation conditions was in the robot's conflict response, rather than other extraneous task-related trajectories such as reaching, pressing of the liquid pumps.

Upon detection of a conflict, the LDS moved the target location of the DS to the desired KD behind the current location of the robot. This effectively moved the robot backwards by KD before the upper layer module moved the target location of the DS again to the dispenser button for a reattempt to access the resource. The number of RA shown in the robot's motions were programmed by changing the number of times the LDS was used before switching the trajectory generation to a quintic spline to the robot's starting position.

\subsection{Analysis}

This experiment was structured as a non-standard, two-factor, factorial design where variables $\mathrm{KD}$ and RA serve as the two crossed factors. The five levels $(0,1,2,3,4)$ of RA are factored with the four levels of $\operatorname{KD}(0,10,19,40)$. Two elements of this experiment structure make this factorial design non-standard. First, none of the values of KD and RA apply to the control condition, requiring the analysis to include a control group outside the KD-RA factorial. Second, there are some combinations of KD-RA that are missing from the experiment due to the reasons described earlier. 
While this is not ideal, subjecting the participants to a smaller number of conditions shortened the length of the within-subjects study, thereby avoiding potential effects of pencil-whipping.

To test the aforementioned hypotheses, the responses to the six measures from the survey are analyzed using a regression analysis with a linear mixed effects model. ${ }^{9}$ Keeping in mind the repeated measures aspect of this study (all participants saw all 15 videos), Participant was used as a random factor and $\mathrm{KD}$ and $\mathrm{RA}$ as fixed factors. In this analysis, the mean responses to the $(\mathrm{KD}=0$, $\mathrm{RA}=0$ ) pair, $\mu_{0}$, is used as the reference point with which the responses to the remaining parameter pairs are modelled:

$$
\begin{gathered}
(K D=0, R A=0) \sim \mu_{0} \\
\text { Smooth_Stop } \sim \mu_{0}+\tau_{\text {Smooth_Stop }}, \\
(K D=\alpha, R A=0) \sim \mu_{0}+K D_{\alpha}, \\
(K D=\alpha, R A=\beta) \sim \mu_{0}+K D_{\alpha}+K D_{\alpha}: R A_{\beta} .
\end{gathered}
$$

Here, $\tau_{\text {Smooth_Stop }}$ refers to the difference in survey response between the Smooth Stop and the $(\mathrm{KD}=0, \mathrm{RA}=0)$ condition; $K D_{\alpha}$ refers to the main effect of $K D=\alpha \in[10,19,40]$ compared to $\mathrm{KD}=0$ where $\alpha$ is one of the non-zero $\mathrm{KD}$ levels tested; $R A_{\beta}$ refers to the main effect of $R A=\beta \in$ $[1,2,3,4]$ compared to $\mathrm{RA}=0$, where $\beta$ is one of the non-zero RA levels tested; $K D_{\alpha}: R A_{\beta}$ is the interaction effect between $K D_{\alpha}$ and $R A_{\beta}$. This approach allows us to identify the effects of the NHG conditions against the control condition while taking into account the main effects of $\mathrm{KD}$ and RA. All of the statistical analyses used a significance level of $\alpha=.05$. The Tukey method was used to conduct post-hoc analyses.

\subsection{Results}

A total of 50 people participated in the study (16 females, 33 males, 1 preferred not to disclose). On average, it took approximately 18 minutes for the participants to complete the survey $(M=18: 27$, $S D=9: 03)$. The age of the participants ranged from 21 to $52(M=32, S D=7.0)$. We present the results in the order of the questionnaire measures except for the Usefulness measure, which was the only one to fail to meet the internal reliability standard and has a Chronbach's $\alpha<0.70$.

6.4.1 Hesitancy. Results support Hypothesis 1 and demonstrate that the NHG-generated robot motions are perceived to be significantly more hesitant than that of the control condition $(F(1,692)=30.4, p<0.001)$. There are significant differences in Hesitancy across the different values of $\operatorname{KD}(F(3,692)=3.98, p<0.01)$ and $\operatorname{RA}(F(4,692)=7.80, p<0.001)$. The interaction between main effects of $\mathrm{KD}$ and RA is negligible $\left(X^{2}(6)=10.5, p=0.11\right)$. We find that female participants rated robots to be more hesitant than males $(Z=-2.89, p<0.01)$, with Gender being a significant factor in the perception of Hesitancy $(F(2,692)=6.50, p<0.01)$.

We hypothesized that robot hesitation responses generated with a human-inspired kickback distance $(0 \leq K D<19 \mathrm{~mm})$ would be perceived as being more hesitant than those with higher values of $\mathrm{KD}$ (Hypothesis 4). While post-hoc analyses indicate that there are significant differences on Hesitancy among the human-inspired values of $\mathrm{KD}$, we do not find support for the hypothesis. The robot motions generated with $\mathrm{KD}=0$ are perceived to convey a significantly higher level of Hesitancy than those with $\mathrm{KD}=10$ and $\mathrm{KD}=19$ (see Figure 8). This is interesting given that the

\footnotetext{
${ }^{9}$ A Multi-level Modelling (MLM) using the lme4 package in R with Restricted Maximum Likelihood (REML) was used for model fitting. Given that the structure of this study is a non-standard two-factor factorial design, a more familiar statistical modeling such as Analysis of Variance (ANOVA) cannot be used. However, Multi-level Modelling (MLM) allows for data from such non-standard study designs to be captured such that inferential statistics can still be conducted.
} 




Error Bars: $95 \% \mathrm{Cl}$

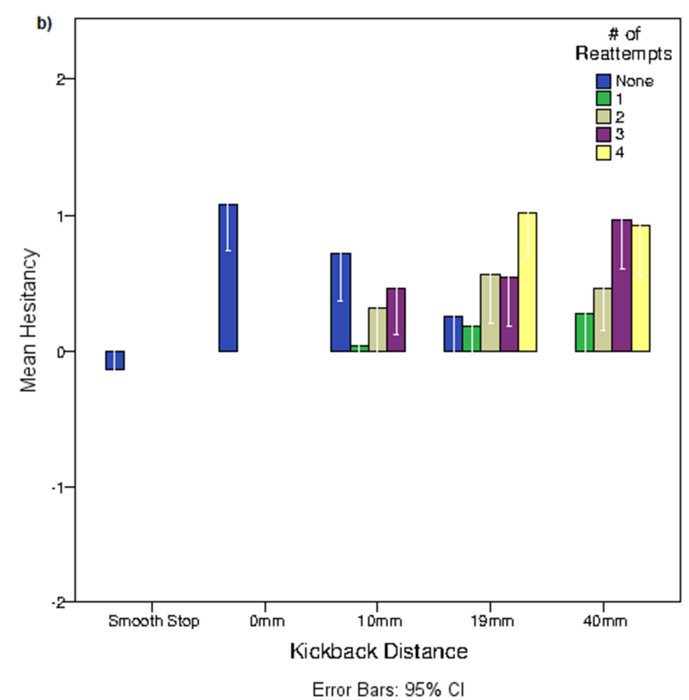

Fig. 8. The perceived Hesitancy scores (a 5-point Likert scale, ranging from Not Hesitant (-2) to Hesitant (+2)) collected for the different levels of KD and RA. Analyses of the scores across all NHG-generated motions suggest that there are significant differences in Hesitancy among the human-inspired values of KD.

robot does not perform any re-attempts in the $(\mathrm{KD}=0, \mathrm{RA}=0)$ condition-the only condition with a value of $\mathrm{KD}=0$-and, therefore, is the least negotiative of all NHG conditions.

6.4.2 Persistency. We find empirical support that the NHG-generated robot motions are perceived to be significantly more persistent than smooth stop motions $(F(1,686)=16.3, p<0.001)$. As shown in Figure 9, the Persistency score is higher for $\mathrm{KD}=[10,19]$ for most non-zero RA values than that of the control condition or the reference $(\mathrm{KD}=0, \mathrm{RA}=0)$ condition, although this is not true for all values of RA.

We also find a significant interaction effect in Persistency scores between KD and RA $\left(X^{2}(6)=\right.$ $12.65, p<0.05)$. Hence, the results do not offer straight-forward support for Hypothesis 5 that robot hesitation responses showing a higher number of RA are perceived to be more persistent than those with lower RA values.

6.4.3 Animacy and Anthropomorphism. Results of the analysis on Animacy and Anthropomorphism support Hypothesis 2: the NHG-generated motions are perceived to be more animate and anthropomorphic than the smooth stopping motions in the control condition (Animacy: $F(1,692)=11.9, p<0.001$; Anthropomorphism: $F(1,692)=10.4, p<0.01)$. Figure 10 presents the perceived Animacy and Anthropomorphism scores. However, it is puzzling to note that Animacy and Anthropomorphism scores overall are low. This may be because the video showed reaching motions of a robot contrast with that of a person next to it, the latter of which may have attenuated the perceived Animacy and Anthropomorphism of the robot's motion.

6.4.4 Dominance. Contrary to Hypothesis 3, the results suggest that the NHG-generated motions are perceived to be significantly less dominant than the smooth stopping motions of the control condition $(F(1,686)=35.2, p<0.001)$. We also find that the male participants rated the robot's motions to be more dominant than the females did $\left(X^{2}(2)=6.55, p<0.05\right)$. There is a significant interaction effect between the main effects of $\mathrm{KD}$ and $\mathrm{RA}\left(X^{2}(6)=31.6, p<0.001\right)$. Hence, the effects of factors KD and RA cannot be interpreted independently from each other, and 


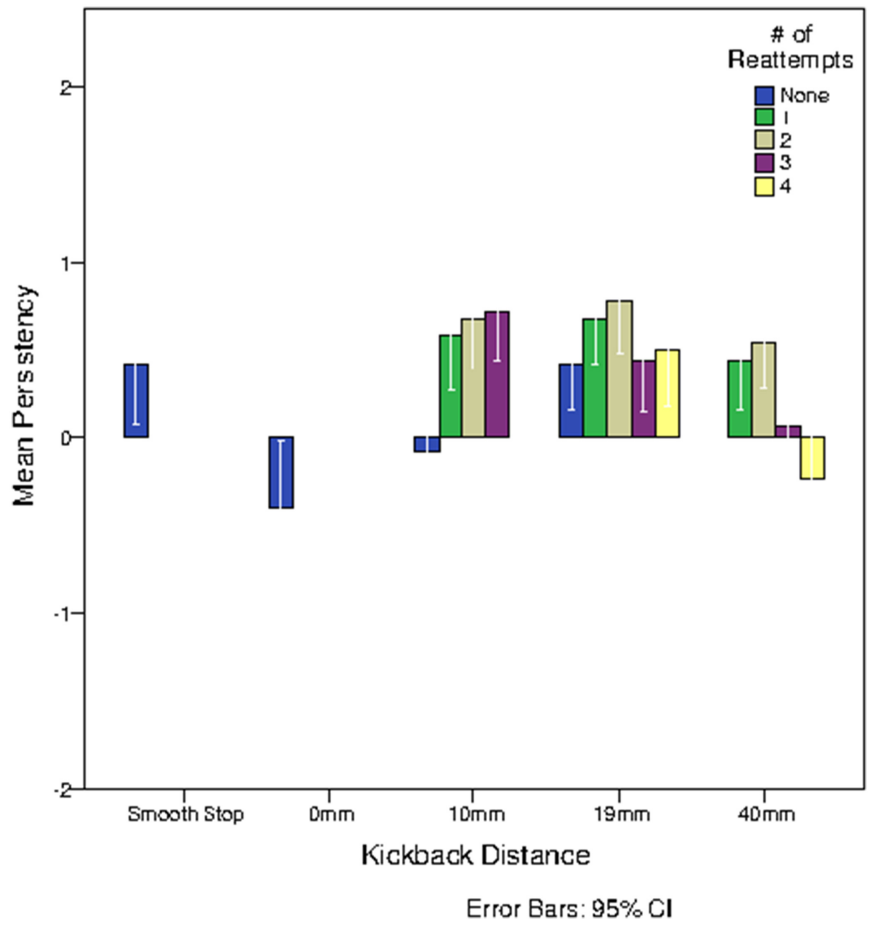

Fig. 9. Perceived Persistency-ranging from Not Persistent (-2) to Persistent (+2)-of different KD by RA values. There is a significant interaction effect between KD and RA. Nonetheless, NHG-generated motions are perceived to be significantly more persistent than the motion implemented in the Smooth Stop condition.
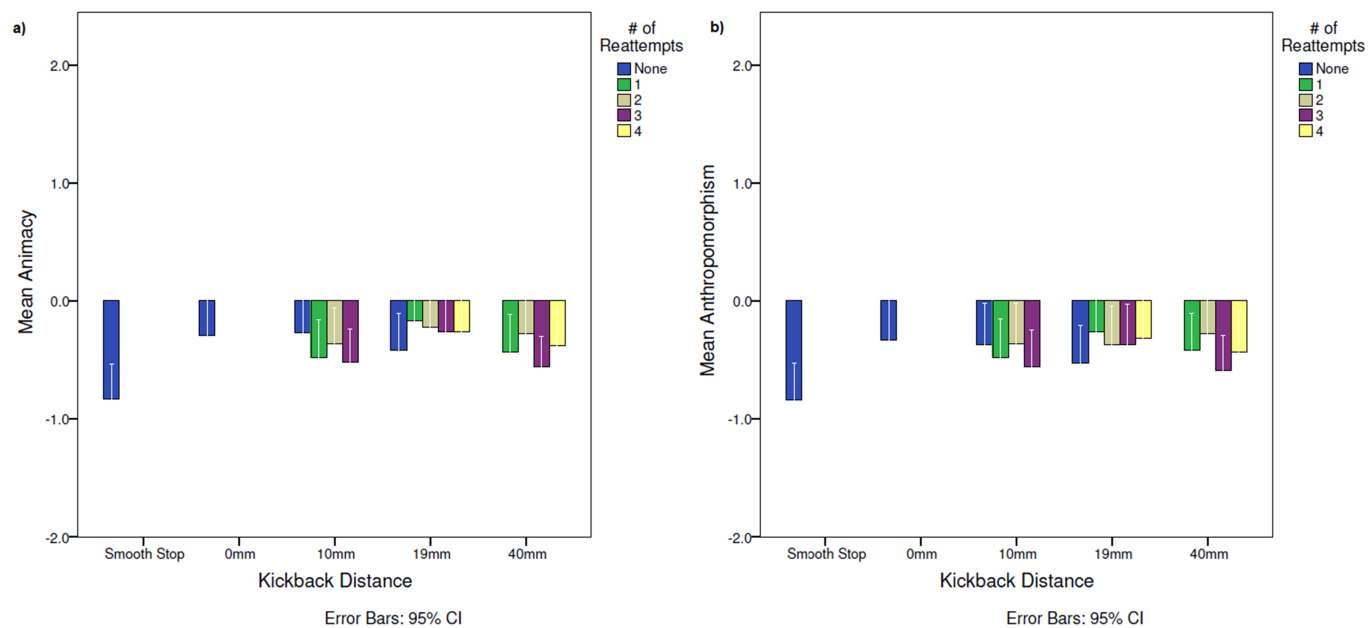

Fig. 10. (a) Perceived Animacy of different KD by RA values. The NHG-generated behaviours are, in general, perceived to be more animate than that smooth stopping behaviour. (b) Perceived Anthropomorphism of different KD by RA values. Echoing the results of Animacy, NHG-generated motions are perceived to be more anthropomorphic than the smooth stopping behaviours of the robot. 


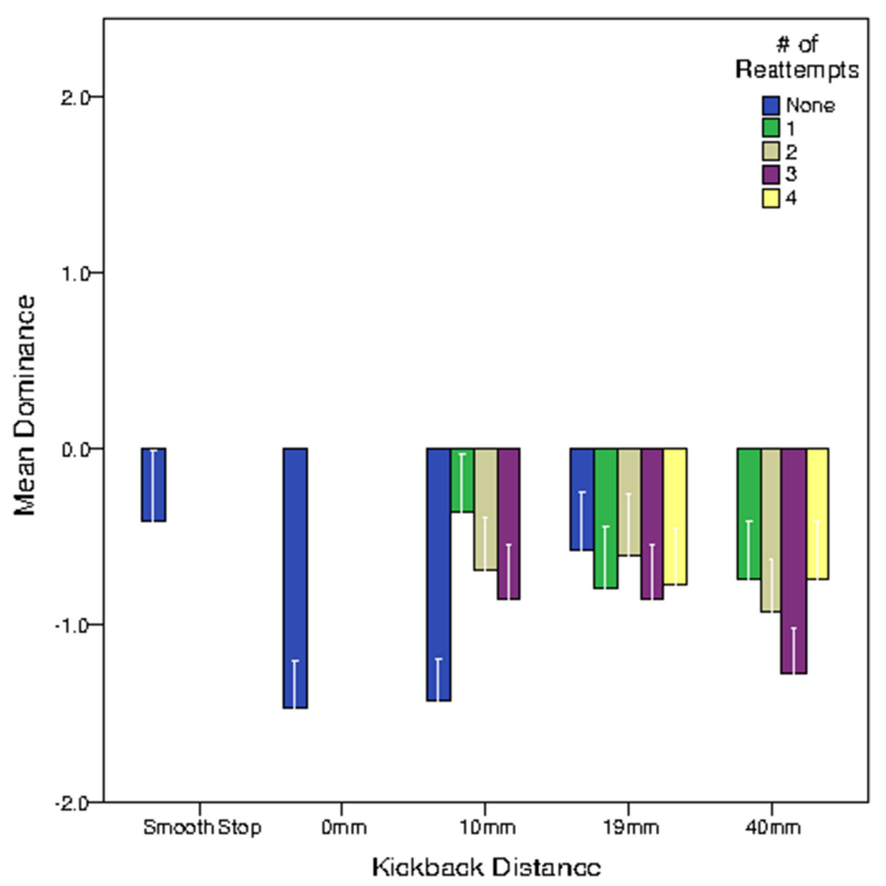

Error Bars: $95 \% \mathrm{Cl}$

Fig. 11. Perceived Dominance of different KD by RA values. Contrary to Hypothesis 3, NHG-generated motions were perceived to be less dominant than the smooth stopping alternative.

support for Hypothesis 5 remains inconclusive. See Figure 11 for the distribution of the Dominance measure.

6.4.5 Qualitative Feedback. Keeping in mind the principles of grounded theory, two experimenters coded the open text entries participants optionally submitted. Only $50 \%$ of the participants provided at least one adjective throughout the survey. The responses captured using an open text field were mostly adjectives, as requested in the questionnaire. Most of these participants submitted responses to four or fewer conditions. Given the small number of responses collected, our qualitative analysis is presented only as a means to complement the quantitative findings discussed above.

The types of adjectives that participants used fell into approximately three categories: adjectives describing the perceived expression or conveyed personality of the robot (e.g., hesitant, confident, scared), motion quality of the robot (e.g., smooth, jerky), perceived anthropomorphism (e.g., humanlike, calculated, artificial), and perceived performance of the robot (e.g., efficient, smart). In all of the conditions, one of the words "hesitant" and "persistent" was mentioned by at least one participant in all but condition $(\mathrm{KD}=40, \mathrm{RA}=4)$.

When the participant responses to Smooth Stop and $(\mathrm{KD}=0, \mathrm{RA}=0)$ are compared, both of the robot motions are described as hesitant. However, whereas the Smooth Stop condition is described with words hinting at low anthropomorphism (e.g., awkward, cautious, mechanical and robotic), motion quality of $(\mathrm{KD}=0, \mathrm{RA}=0)$ is described with positive adjectives such as safe and smart. The Smooth Stop condition is described as expressing a sense of dominance (e.g., aggressive and assertive), whereas the $(\mathrm{KD}=0, \mathrm{RA}=0)$ condition is described to be apprehensive and wary, despite being responsive (e.g., pushover, scared, submissive). Based on the quantitative results mentioned 
above, the $(\mathrm{KD}=0, \mathrm{RA}=0)$ condition received the lowest mean Persistency and Dominance scores, while scoring highly on the Hesitancy measure. The qualitative description of the robot's motion in this condition supports and complements these results. The Smooth Stop condition also received the lowest Animacy and Anthropomorphism scores, which is corroborated in the qualitative finding.

In comparison, the participants perceived the NHG-generated motions with $\mathrm{KD}=10$ and 19 to be efficient, calculated, and cautious. The robot motions with $\mathrm{KD}=19$, in particular, were described as more life-like and aware across the RA values, except for $\mathrm{RA}=4$, which was described to be submissive and unsure. The NHG-generated motions with $\mathrm{KD}=40$ were described as timid and shy. This affirms the low Dominance scores collected for these conditions. In addition, $\mathrm{KD}=40$ motions with lower RA values were perceived as compromising and slow, while those with higher RA values were seen as scared.

\section{DISCUSSION}

The purpose of this work was to design and validate the efficacy of a trajectory generator robots can use to exhibit human recognizable robot hesitation behaviours for possible nonverbal HR negotiations. To this end, the human hesitation trajectories collected and labelled in Study 1 was essential to identifying the characteristic features necessary to create human recognizable hesitations. The results from Study 1 also informed the design of the NHG and the values of its parameters. Study 2 results demonstrate that the NHG-generated motions are indeed distinguished from smooth stopping behaviours of a robot. The motions produced by the NHG were perceived to be more hesitant and persistent. They were also observed to be more animate and anthropomorphic. These results provide empirical support for using the NHG for in-person HRI where human behavioural responses to the artificial hesitations can be observed. However, there were also surprising findings.

First, counterintuitively, the $(\mathrm{KD}=0, \mathrm{RA}=0)$ condition received the highest Hesitancy score. The qualitative understanding of the participants' perception of the motion offers some explanation for this finding (i.e., the robot motion with this parameter setting was considered to constitute a highly apprehensive and hesitant behaviour). Moreover, this condition received the lowest Persistency score. This is consistent with the concept of persistency, in which a higher value of RA is more likely to be considered to exhibit a higher Persistency. This interpretation does not generalize across all levels of $\mathrm{KD}$, as demonstrated by the significant interaction effect of $\mathrm{KD}$ and $\mathrm{RA}$ observed in the Persistency scores. Given that this condition did not include any re-attempt behaviours and, therefore, was non-negotiative in nature, it does not make sense to use this set of parameters to investigate in-person HR non-verbal negotiations. However, the human-inspired KD values, 10 and 19, received high Persistency scores across the various RA values. Moreover, taking into account variabilities by RA values, $K D=19$ received a relatively high level of Hesitancy in comparison to other values of $\mathrm{KD}$ in general. This also coincides with the average $\mathrm{KD}$ value discovered in $\mathrm{HH}$ hesitations (see Section 4.2), and confirms the efficacy of using this value for generating artificial hesitations with the NHG.

Second, contrary to Hypothesis 3, the motions produced by the NHG are perceived to be less dominant than that of the smooth stopping motions. This is surprising, because the smooth stopping motion employed in this study only had a short pausing behaviour before the robot retracted back. This is in contrast to motions generated by the NHG with non-zero RA values that exhibited multiple back-and-forth motions that could be perceived as more dominant than a pause. In observing the distribution of Dominance scores, in general, motions with a high RA value are seen to be less dominant than those with a lower RA-although this trend is not significant. This result suggests the possibility that the resulting negotiative hesitation behaviours designed to exhibit multiple RA are perceived to be timid and apprehensive. 


\subsection{Limitations}

It is also important to note the limitations of the studies presented. First, due to the limitation of the videos available, Hypothesis 1 could not be fully tested based on the data collected. Second, since Study 2 is only a video-based study, we cannot generalize the results of this study to possible user perception of or behavioural reaction to the NHG-based hesitations in in-person HRI. Therefore, we conducted a controlled in-person HRI experiment to investigate the real-time efficacy of the NHG generated hesitation behaviours with positive results. As the follow-up experiment is outside the scope of this article, we do not report the results here. However, the details of the experiment and related results are available in Reference [26].

Third, industrial robots today are not typically equipped with motion capture systems to monitor individual motions. It is also the case that many robot manipulators use trapezoidal trajectory profiles to generate motion instead of the humanlike quintic splines and LDS discussed in this article. Therefore, it may be unrealistic to expect the proposed NHG to be implemented in industrial manufacturing environments today. However, as collaborative robotics becomes more commonplace in the near future demanding more intuitive and humanlike communication cues to be implemented, we believe the highly reactive, communicative gestures, such as those produced using NHG, will prove to be effective in real-life.

\section{CONCLUSION}

In this article, we presented NHG, a trajectory generator with which one can investigate the role robot negotiative hesitations can play in an interactive HR resolution of resource conflicts. To this end, naturally occurring negotiative hesitations in humans were collected from an HHI experiment (Study 1). A video-based online study involving 300 participants helped rate each sample of hesitations on its expressed level of Hesitancy and Persistency. Exploration of the numerous features of the human hesitation trajectories revealed that, unlike R-type hesitation behaviours investigated in our previous work, negotiative hesitations take into consideration the motions of both agents. This led to the discovery of hesitation loops, which are trajectory patterns found in the majority of the negotiative hesitation samples collected in Study 1. The design of the NHG was inspired by the hesitation loop trajectory patterns. It was designed such that the change in the relative distance between the two interacting agents to the shared target is used to determine when the robot should trigger its hesitation behaviour.

In implementing the NHG for in-person HRI, it is important that the trajectory output of the NHG be established as communicative of hesitation. Study 2 helped validate the efficacy of the NHG-based hesitation behaviours. It provides empirical evidence that robot motions generated by the NHG are perceived to be more hesitant, animate, and anthropomorphic than those of a smooth stopping behaviour of the same robot.

This work contributes to a better understanding of negotiative hesitations naturally found in $\mathrm{HHI}$ and provides a validated trajectory generator with which one can use to explore HR nonverbal negotiation of resource conflicts. We plan to take the first step in investigating such in-person HRI using NHG-generated robot hesitations.

\section{REFERENCES}

[1] Henny Admoni, Anca Dragan, Siddhartha S. Srinivasa, and Brian Scassellati. 2014. Deliberate delays during robotto-human handovers improve compliance with gaze communication. In Proceedings of the ACM/IEEE International Conference on Human-robot Interaction. ACM, 49-56. DOI : https://doi.org/10.1145/2559636.2559682

[2] Amazon.com. 2016. Amazon Mechanical Turk: Artificial Artificial Intelligence. Retrieved from https://www.mturk. com/mturk/welcome.

[3] Michael Argyle. 1991. Cooperation: The Basis of Sociability. Routledge, London/New York. 288 pages. Retrieved from http://www.amazon.com/Cooperation-Basis-Sociability-Michael-Argyle/dp/0415035465. 
[4] Christoph Bartneck, Dana Kulić, Elizabeth Croft, and Susana Zoghbi. 2009. Measurement instruments for the anthropomorphism, animacy, likeability, perceived intelligence, and perceived safety of robots. Int. F. Soc. Robot. 1, 1 (Nov. 2009), 71-81. DOI : https://doi.org/10.1007/s12369-008-0001-3

[5] Christoph Bartneck, Michel van der Hoek, Omar Mubin, and Abdullah Al Mahmud. 2007. "Daisy, Daisy, give me your answer do!" In Proceedings of the ACM/IEEE international conference on Human-robot interaction. ACM Press, 217. DOI : https://doi.org/10.1145/1228716.1228746

[6] Crystal Chao and Andrea Thomaz. 2013. Controlling social dynamics with a parametrized model of floor regulation. f. Hum. Robot Interact. 2, 1 (2013), 4-29. DOI : https://doi.org/10.5898/jhri.2.1.chao

[7] Martin Corley and Oliver W. Stewart. 2008. Hesitation disfluencies in spontaneous speech: The meaning of um. Lang. Linquist. Compass 2, 4 (July 2008), 589-602. DOI : https://doi.org/10.1111/j.1749-818X.2008.00068.x

[8] Christian Dondrup, Nicola Bellotto, and Marc Hanheide. 2014. Social distance augmented qualitative trajectory calculus for human-robot spatial interaction. In Proceedings of the 23rd IEEE International Symposium on Robot and Human Interactive Communication. 519-524. DOI : https://doi.org/10.1109/ROMAN.2014.6926305

[9] Christian Dondrup, Christina Lichtenthäler, and Marc Hanheide. 2014. Hesitation signals in human-robot head-on encounters: A pilot study. In Proceedings of the 9th ACM/IEEE International Conference on Human-Robot Interaction. 154-155. DOI : https://doi.org/10.1145/2559636.2559817

[10] Leonard William Doob. 1990. Hesitation: Impulsivity and Reflection. Greenwood Press, Westport, CT. 214 pages. Retrieved from http://books.google.com/books?id=Q7B9AAAAMAAJ\&pgis=1 http://www.questia.com/read/27488222.

[11] Nadia Figueroa, Ana Lucia Pais Ureche, and Aude Billard. 2016. Learning complex sequential tasks from demonstration: A pizza dough rolling case study. In Proceedings of the 11th ACM/IEEE International Conference on Human-Robot Interaction. ACM/IEEE, 611-612. DOI : https://doi.org/10.1109/HRI.2016.7451881

[12] Kerstin Fischer, Oliver Niebuhr, Lars C. Jensen, and Leon Bodenhagen. 2019. Speech melody matters-How robots profit from. Trans. Hum. Robot Interact. 9, 1 (2019), 1-21.

[13] Tamar Flash and Neville Hogan. 1985. The coordination of arm movements: Mathematical model. f. Neurosci. 5, 7 (1985), 1688-1703. Retrieved from http://www.jneurosci.org/cgi/content/abstract/5/7/1688.

[14] K. Gillespie-Lynch, P. M. Greenfield, Y. Feng, S. Savage-Rumbaugh, and H. Lyn. 2013. A cross-species study of gesture and its role in symbolic development: Implications for the gestural theory of language evolution.Front. Psychol. 4 (Jan. 2013), 160. DOI : https://doi.org/10.3389/fpsyg.2013.00160

[15] David B. Givens. 2002. The Nonverbal Dictionary of Gestures, Signs \& Body Language Cues. Center for Nonverbal Studies Press, Spokane, Washington. Retrieved from http://www.mikolaj.info/edu/Body_Language_-_List_of_Signs_ n_Gestures.pdf.

[16] Roberta Michnick Golinkoff. 1986. "I beg your pardon?”: The preverbal negotiation of failed messages. f. Child. Lang. 13, 03 (Sep. 1986), 455-476. DOI : https://doi.org/10.1017/S0305000900006826

[17] R. M. Golinkoff. 1993. When is communication a meeting of minds? J. Child. Lang. 20, 1 (Feb. 1993), 199-207. Retrieved from http://journals.cambridge.org/abstract_S030500090000920X; http://www.ncbi.nlm.nih.gov/pubmed/8454683.

[18] Bidan Huang, Miao Li, Ravin Luis De Souza, Joanna J. Bryson, and Aude Billard. 2016. A modular approach to learning manipulation strategies from human demonstration. Auton. Robots 40, 5 (2016), 903-927. DOI : https://doi.org/10.1007/ s10514-015-9501-9

[19] Tanaka Kazuaki, Ozeki Motoyuki, and Oka K. A. Natsuki. 2010. The hesitation of a robot: A delay in its motion increases learning efficiency and impresses humans as teachable. In Proceedings of the ACM/IEEE International Conference on Human-Robot Interaction, Vol. 8821007. IEEE, 189-190. DOI : https://doi.org/10.1109/HRI.2010.5453200

[20] S. Mohammad Khansari-Zadeh and Aude Billard. 2011. Learning stable non-linear dynamical systems with Gaussian mixture models. IEEE Trans. Robot. 27, 5 (2011), 943-957. DOI : https://doi.org/10.1109/TRO.2011.2159412

[21] Seungsu Kim, Ashwini Shukla, and Aude Billard. 2014. Catching objects in flight. IEEE Trans. Robot. 30, 5 (2014), 1-17. DOI : https://doi.org/10.1109/TRO.2014.2316022

[22] David A. Leavens, Jamie L. Russell, and William D. Hopkins. 2005. Intentionality as measured in the persistence and elaboration of communication by chimpanzees (Pan troglodytes). Child Dev. 76, 1 (2005), 291-306. http://onlinelibrary. wiley.com/doi/10.1111/j.1467-8624.2005.00845.x/full

[23] James G. Martin and Winifred Strange. 1968. The perception of hesitation in spontaneous speech. Percept. Psychol. 3 , 6 (1968), 427-438. DOI : https://doi.org/10.3758/BF03205750

[24] Sandra Merlo and Plínio Almeida Barbosa. 2010. Hesitation phenomena: A dynamical perspective.Cogn. Process. 11, 3 (Aug. 2010), 251-61. DOI : https://doi.org/10.1007/s10339-009-0348-x

[25] Seyed Mohammad Khansari-Zadeh and Aude Billard. 2012. A dynamical system approach to realtime obstacle avoidance. Auton. Robots 32, 4 (2012), 433-454. DOI : https://doi.org/10.1007/s10514-012-9287-y

[26] AJung Moon. 2017. Negotiating with Robots: Meshing Plans and Resolving Conflicts in Human-Robot Collaboration. Doctor of Philosophy-PhD thesis, University of British Columbia. DOI : https://doi.org/10.14288/1.0348225 
[27] AJung Moon, Chris A. C. Parker, Elizabeth A. Croft, and H. F. Machiel Van der Loos. 2011. Did you see it hesitate?Empirically grounded design of hesitation trajectories for collaborative robots. In Proceedings of the IEEE/RSF International Conference on Intelligent Robots and Systems. IEEE/RSJ, 1994-1999. DOI : https://doi.org/10.1109/IROS.2011. 6048228

[28] AJung Moon, Chris A. C. Parker, Elizabeth A. Croft, and H. F. Machiel Van der Loos. 2013. Design and impact of hesitation gestures during human-robot resource conflicts. f. Hum. Robot Interact. 2, 3 (Sep. 2013), 18-40. DOI : https: //doi.org/10.5898/JHRI.2.3.Moon

[29] Youngme Moon and Clifford Nass. 1996. How "Real" are computer personalities?: Psychological responses to personality types in human-computer interaction. Commun. Res. 23, 6 (Dec. 1996), 651-674. DOI : https://doi.org/10.1177/ 009365096023006002

[30] Allan Netick and Stuart T. Klapp. 1994. Hesitations in manual tracking: A single-channel limit in response programming.f. Exp. Psychol. Hum. 20, 4 (1994), 766-782. DOI : https://doi.org/10.1037/0096-1523.20.4.766

[31] Y. Ogai and T. Ikegami. 2008. Microslip as a simulated artificial mind. Adapt. Behav. 16, 2-3 (Apr. 2008), $129-147$. DOI : https://doi.org/10.1177/1059712308089182

[32] Ana-Lucia Lucia Pais Ureche and Aude Billard. 2015. Learning bimanual coordinated tasks from human demonstrations. In Proceedings of the 10th Annual ACM/IEEE International Conference on Human-Robot Interaction Extended Abstracts. 141-142. DOI : https://doi.org/10.1145/2701973.2702007

[33] Morgan Quigley, Ken Conley, Brian P. Gerkey, Josh Faust, Tully Foote, Jeremy Leibs, Rob Wheeler, and Andrew Y. Ng. 2009. ROS: An open-source robot operating system. In Proceedings of IEEE International Conference on Robotics and Automation-Workshop on Open Source Software. IEEE. Retrieved from http://www.willowgarage.com/sites/default/ files/icraoss09-ROS.pdf.

[34] Robert W. Reeder and Roy A. Maxion. 2006. User interface defect detection by hesitation analysis. Proceedings of the International Conference on Dependable Systems and Networks. 61-70. DOI : https://doi.org/10.1109/DSN.2006.71

[35] Peter Rober. 2002. Some hypotheses about hesitations and their nonverbal expression in family therapy practice. 7 . Fam. Ther. 24, 2 (2002), 187-204. DOI : https://doi.org/10.1111/1467-6427.00211

[36] Joaquin F. Sousa-Poza and Robert Rohrberg. 1977. Body movement in relation to type of information (person- and nonperson-oriented) and cognitive style (field dependence) 1. Hum. Commun. Res. 4, 1 (Sep. 1977), 19-29. DOI : https: //doi.org/10.1111/j.1468-2958.1977.tb00592.x

[37] Chikako Suda and Josep Call. 2006. What does an intermediate success rate mean? An analysis of a Piagetian liquid conservation task in the great apes. Cognition 99, 1 (Feb. 2006), 53-71. DOI : https://doi.org/10.1016/j.cognition.2005. 01.005

[38] Rik Van den Brule, Gijsbert Bijlstra, Ron Dotsch, Pim Haselager, and Daniel H. J. Wigboldus. 2016. Warning signals for poor performance improve human-robot interaction. J. Hum. Robot Interact. 5, 2 (2016), 69. DOI: https://doi.org/ 10.5898/jhri.5.2.van_den_brule

[39] Alessandro Vinciarelli, Maja Pantic, and Hervé Bourlard. 2009. Social signal processing: Survey of an emerging domain. Image Vis. Comput. 27, 12 (Nov. 2009), 1743-1759. DOI : https://doi.org/10.1016/j.imavis.2008.11.007

[40] Tomoyuki Yokoi and Kenji Fujisaki. 2009. Hesitation behaviour of hoverflies Sphaerophoria spp. to avoid ambush by crab spiders.Sci. Nat. 96, 2 (Feb. 2009), 195-200. DOI : https://doi.org/10.1007/s00114-008-0459-8

Received September 2019; revised May 2020; accepted July 2020 